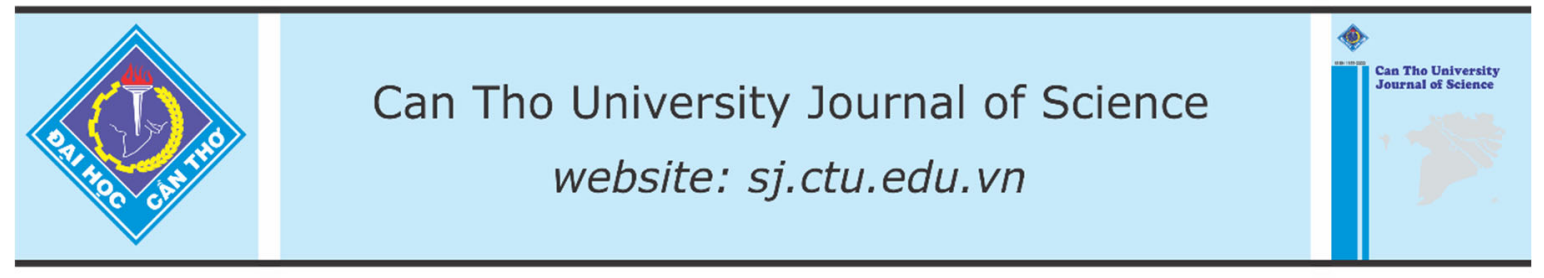

DOI: $10.22144 / c t u . j e n .2019 .013$

\title{
Law of large number for one dimensional Markov process
}

Lam Hoang Chuong ${ }^{1 *}$, Le Thi My Xuan ${ }^{1}$, Nguyen Thi Thu $\mathrm{Ha}^{2}$ and Le Nguyen Thuy Van ${ }^{3}$

${ }^{I}$ College of Natural Sciences, Can Tho University, Vietnam

${ }^{2}$ Master Student in Probability Theory and Statistics - Course 23, Can Tho University, Vietnam

${ }^{3}$ Bachelor Student in Applied Mathematics - Course 40, Can Tho University, Vietnam

*Correspondence: Lam Hoang Chuong (email: lhchuong@ctu.edu.vn)

\section{Article info.}

Received 28 May 2018

Revised 20 Oct 2018

Accepted 29 Mar 2019

\section{Keywords}

Law of large numbers, Markov process, rate of convergence

\section{ABSTRACT}

The aim of this paper is to study the model of Markov process with the state space $\mathbb{Z}$. The applied method is similar to Depauw et al. (2009) and Lam (2014) to prove that this stochastic process converges in probability to a constant (Theorem 1.1) and to give its rate also (Theorem 3.1). Precisely, let $L$ be the corresponding operator of the previous stochastic process and $f$ be a given function, we solve the equation $L g \equiv f$ and then treat the limit of its solutions, the rate of the convergence is instantly given by the convergence of the moment of Markov process.

Cited as: Chuong, L.H., Xuan, L.T.M., Ha, N.T.T. and Van, L.N.T., 2019. Law of large number for one dimensional Markov process. Can Tho University Journal of Science. 11(1): 95-99.

\section{INTRODUCTION}

Let $\left(X_{t}\right)_{t \geq 0}$ be a Markov process on the state space $\mathbb{Z}$ and suppose that operators $\left\{P_{t}\right\}_{t \geq 0}$ acts on a bounded function $f: \mathbb{Z} \rightarrow \mathbb{R}$

$$
P_{t} f(k)=E\left\{f\left(X_{t}\right) \mid X_{0}=k\right\} \text {. (1.1) }
$$

According to Markov chain's property:

$$
P_{t} f(k)=E\left\{f\left(X_{t+s}\right) \mid X_{S}=k\right\},
$$

for all $s \geq 0$.

Let operator $L$ is a derivative of $\left\{P_{t}\right\}_{t \geq 0}$ at $t=0$, i.e., $L f=\lim _{t \rightarrow 0^{+}} \frac{P_{t} f-f}{t}$

if the limit exists. The domain $\mathcal{D}(L)$ of this operator is defined as the set of functions such that the limit exists.
For $f \in \mathcal{D}(L)$, we also have pointwise continuity

$$
L f(k)=\lim _{t \rightarrow 0^{+}} \frac{P_{t} f(k)-f(k)}{t}, \forall k \in \mathbb{Z}
$$

The operator $L$ is called the infinitesimal generator of Markov process $\left(X_{t}\right)_{t \geq 0}$.

Considering Markov process $\left(X_{t}\right)_{t \geq 0}$ with $X_{0}=0$, the conductance of the edge between $[k ; k+1]$ is $\lambda$ and the conductance of the edge between $[k ; k-$ $1]$ is $\gamma$. When $t \rightarrow 0$,

$$
\begin{gathered}
\mathbb{P}\left\{X_{t+s}=k+1 \mid X_{S}=k\right\}=\lambda t+o(t), \\
\mathbb{P}\left\{X_{t+s}=k-1 \mid X_{S}=k\right\}=\gamma t+o(t), \\
\mathbb{P}\left\{X_{t+s}=k \mid X_{S}=k\right\}=1-(\lambda+\gamma) t+o(t) .
\end{gathered}
$$

Also, 
$P_{t} f(k)=E\left\{f\left(X_{t+s}\right) \mid X_{S}=k\right\}=\lambda t f(k+1)+o(t) f(k+1)+\gamma t f(k-1)$ It is also called the weak law of large numbers for $+o(t) f(k-1)+f(k)-(\lambda+\gamma) t f(k)+o(t) f(k)$,

where $\lim _{t \rightarrow t} \frac{o(t)}{t}=0$.

Based on the definition of infinitesimal generator $L$, we have

$L f(k)=\lim _{t \rightarrow 0} \frac{P_{t} f(k)-f(k)}{t}=\lambda f(k+1)+\gamma f(k-1)-(\lambda+\gamma) f(k)$.

In the case $\lambda \neq \gamma$, the process $\left(X_{t}\right)_{t \geq 0}$ is defined as above called unbalanced onedimensionalMarkov process.

Stochastic process model has various applications in real-life situations. That is the increase or decrease ofan individual after a short period of time, which is also called thebirth-death process. In term of a dynamical system, it is the random transfer process of a particle inside a homogeneous conductor. In queueing theory, the number of customers, who just came or left after a short period of time,can be modeled as aMarkov process.

In [2], Lam (2014) introduced a model of Markov process with the conductance of the edge between $[k ; k+1]$ is $\alpha_{k}$, where $\left(\alpha_{k}\right)_{k \in \mathbb{Z}}$ be a stationary process. He also proved the central limit theorem $\frac{X t}{\sqrt{t}} \stackrel{D}{\longrightarrow} N\left(0 ; \sigma^{2}\right)$ as $t \rightarrow+\infty$.

In the above expression, $\stackrel{D}{\longrightarrow}$ denoting convergence in distribution of random variables.

In this paper, we consider the previous model of unbalanced stochastic process $\left(X_{t}\right)_{t \geq 0}$ with the infinitesimal generator $L$ as in (1.2)and initial state $X_{0}=0$. Now we study the law of the process for large enough $t$. In this case, every state of the process is transient(Ross, 2010), and based on this result, we prove the existence of law of large number for the sequence $\left(X_{t}\right)_{t \geq 0}$. We have the following theorem.

Theorem 1.1 For all unbalanced stochastic process $\left(X_{t}\right)_{t \geq 0}$ as above, we get

$$
\frac{X_{t}}{t} \stackrel{P}{\longrightarrow} \lambda-\gamma
$$

As $t \rightarrow+\infty$. In the above expression, $\stackrel{P}{\longrightarrow}$ denoting convergence in probability of random variables and $G=\lambda-\gamma$ is the result of limit. 


\section{MAIN RESULTS}

We now consider the unbalanced stochastic process introduced in Section 1. In this section, we take into account the case $\lambda>\gamma$ while the proof for the case $\lambda<\gamma$ can be treated by the same argument. The main result regardingtherate of convergencefor the law of large number can be presented as follow.

\section{Theorem 3.1 We have}

$$
d\left(\frac{X_{t}}{t}, G\right)=O\left(t^{-1}\right)
$$

Here, by definition, the function $f(t)=O(g(t))$ if $\limsup |f(t) / g(t)|<\infty$.

Under the view of law of large numbers, the simplest case is the independent and identically distributed variables (i.i.d).There are two different types: Weak and strong law of large numbers. In our model, the concerning stochastic process is a Markov process which is more general than the case i.i.d. Moreover, we also illustrate the law of large numbers for dependent variablesand evaluate the rate of convergence.

We have the following necessary lemmas:

Lemma 3.1 Let $\varphi: \mathbb{Z} \rightarrow \mathbb{R}$ be a given function, there exists a unique function $\Phi: \mathbb{Z} \rightarrow \mathbb{R}$ such that

$\left\{\begin{array}{c}\mathrm{L} \Phi \equiv \varphi \\ \Phi(0)=0\end{array}\right.$

Proof.

It is easy to find $\Phi(0)$.

For all $m$, we consider the equation $L \Phi(m)=$ $\varphi(m)$.

$\mathrm{L}$ is an operator defined as above, we have

$$
\begin{gathered}
\lambda \Phi(m+1)+\gamma \Phi(m-1)-(\lambda+\gamma) \Phi(m) \\
=\varphi(m) .
\end{gathered}
$$

In other words,

$$
\begin{aligned}
\Phi(m+1)-\Phi(m) & \\
& =\frac{\gamma}{\lambda}[\Phi(m)-\Phi(m-1)] \\
& +\frac{1}{\lambda} \varphi(m) .
\end{aligned}
$$

Let $\rho=\frac{\gamma}{\lambda}$, we obtain

$$
\begin{aligned}
& \Phi(m+1)-\Phi(m) \\
& \quad=\rho[\Phi(m)-\Phi(m-1)] \\
& \quad+\frac{1}{\lambda} \varphi(m) .
\end{aligned}
$$

By recursion on $\mathrm{m}$, it yields

$$
\Phi(m+1)-\Phi(m)=\frac{1}{\lambda} \sum_{k=0}^{+\infty} \rho^{k} \varphi(m-k) .
$$

Then we divide the equations in to two cases according to $m$, and iterate one more time.

In case $m \geq 1$, we find

$$
\Phi(m)=\frac{1}{\lambda} \sum_{l=0}^{m-1} \sum_{k=0}^{+\infty} \rho^{k} \varphi(l-k) .
$$

Similarly, when $m \leq-1$, we also find

$$
\Phi(m)=-\frac{1}{\lambda} \sum_{l=2}^{-m+1} \sum_{k=0}^{+\infty} \rho^{k} \varphi(1-l-k) .
$$

The function $\Phi$ is therefore theunique solution of (3.1).

We return to Theorem 3.1 and analyze

$$
d\left(\frac{X_{t}}{t}, G\right)=\left|E\left(\frac{X_{t}}{t}-G\right)\right|+\left|E\left(\left(\frac{X_{t}}{t}\right)^{2}-G^{2}\right)\right| \text {. }
$$

As $G=\lambda(1-\rho)$ for all $\rho$, it remains to prove two claims $E\left(\frac{X_{t}}{t}-G\right)=\mathrm{O}\left(t^{-1}\right)$ and $E\left(\left(\frac{X_{t}}{t}\right)^{2}-G^{2}\right)=$ $\mathrm{O}\left(t^{-2}\right)$ to obtain the desired result. These properties are stated in Proposition 3.1 and Proposition 3.2.

Proposition 3.1 Let a sequence of random variables $\left(X_{n}\right)_{n \geq 0}$ be defined as above, we have $E\left(\left(\frac{X_{t}}{t}\right)^{2}-\right.$ $\left.G^{2}\right)=O\left(t^{-2}\right)$.

Proof. We consider the function sequence $f_{k} \geq 0$ with the domain $\mathbb{Z}$ such that

$$
\begin{cases}L f_{k} \equiv f_{k-1}, & k \geq 1 \\ f_{0} \equiv 1, & k \geq 1 .\end{cases}
$$

Applying Lemma 3.1 by substituting $\varphi \equiv f_{0}, \Phi \equiv$ $f_{1}$ yields

If $m \geq 1$

$$
\begin{aligned}
f_{1}(m) & =\frac{1}{\lambda} \sum_{l=0}^{m-1} \sum_{k=0}^{+\infty} \rho^{k} f_{0}(l-k) \\
& =\frac{1}{\lambda} \sum_{l=0}^{m-1} \sum_{k=0}^{+\infty} \rho^{k}=\frac{1}{\lambda} \sum_{l=0}^{m-1} \frac{1}{1-\rho} \\
& =\frac{m}{\gamma(1-\rho)}
\end{aligned}
$$

If $m \leq-1$ 


$$
\begin{aligned}
f_{1}(m)=-\frac{1}{\lambda} \sum_{l=2}^{-m+1} & \sum_{k=0}^{+\infty} \rho^{k} f_{0}(1-l-k) \\
& =-\frac{1}{\lambda} \sum_{l=2}^{-m+1} \sum_{k=0}^{+\infty} \rho^{k}=\frac{m}{\lambda(1-\rho)} .
\end{aligned}
$$

Thus,

$$
f_{1}(m)=\frac{m}{\lambda(1-\rho)}
$$

for all $m$.

Again, we use Lemma 3.1 with $\varphi \equiv f_{1}, \Phi \equiv f_{2}$ to obtain

If $m \geq 1$ then

$$
\begin{aligned}
f_{2}(m) & =\frac{1}{\lambda} \sum_{l=0}^{m-1} \sum_{k=0}^{+\infty} \rho^{k} f_{1}(l-k) \\
& =\frac{1}{\lambda^{2}(1-\rho)} \sum_{l=0}^{m-1} \sum_{k=0}^{+\infty} \rho^{k}(l-k) \\
& =\frac{1}{\lambda^{2}(1-\rho)^{2}}\left(\frac{(m-1) m}{2}-\frac{m \rho}{(1-\rho)}\right) .
\end{aligned}
$$

If $m \leq-1$ then

$$
\begin{gathered}
f_{2}(m)=-\frac{1}{\lambda} \sum_{l=2}^{-m+1} \sum_{k=0}^{+\infty} \rho^{k} f_{1}(1-l-k) \\
=-\frac{1}{\lambda^{2}} \sum_{l=2}^{-m+1} \sum_{k=0}^{+\infty} \rho^{k} \frac{(1-l-k)}{1-\rho} \\
=-\frac{1}{\lambda^{2}(1-\rho)} \sum_{l=2}^{-m+1}\left(\sum_{k=0}^{+\infty} \rho^{k}(1-l)\right. \\
\left.\quad+(-k) \rho \sum_{k=0}^{+\infty} \rho^{k-1}\right) \\
=\frac{1}{\lambda^{2}(1-\rho)^{2}}\left(\frac{(m-1) m}{2}-\frac{m \rho}{(1-\rho)}\right) .
\end{gathered}
$$

We conclude that

$$
f_{2}(m)=\frac{1}{\lambda^{2}(1-\rho)^{2}}\left(\frac{(m-1) m}{2}-\frac{m \rho}{(1-\rho)}\right)
$$

for all $m$.

Thenfor all $m$ and $k \geq 1$, one has $L f_{k}(m)=$ $f_{k-1}(m)$.

Substituting $m$ by $X_{t}$ and evaluating expectation of two sides, $L f_{k}\left(X_{t}\right)=f_{k-1}\left(X_{t}\right)$ for all $t \geq 0$. For every $k=1,2$, we claim that

$$
E\left\{f_{k}\left(X_{t}\right)\right\}=\frac{t^{k}}{k}
$$

Where $f_{k}(0)=0$ according to the definition of $f_{k}$ and $X_{0}=0$ according to the assumption of the stochastic process $X_{t}$. Expression (3.2) is proved by the following way.

When $k=1$ :

Let $h_{1}(t)=E\left\{f_{1}\left(X_{t}\right)\right\}$, we have

$$
\begin{aligned}
E\left\{L f_{1}\left(X_{t}\right)\right\} & =\lim _{s \rightarrow 0} E\left\{\frac{E\left\{f_{1}\left(X_{t+s}\right) \mid X_{t}\right\}-f_{1}\left(X_{t}\right)}{s}\right\} \\
= & \lim _{s \rightarrow 0} E\left\{\frac{f_{1}\left(X_{t+s}\right)-f_{1}\left(X_{t}\right)}{s}\right\} \\
= & \lim _{s \rightarrow 0} \frac{h_{1}(t+s)-h_{1}(t)}{s}=\frac{d h_{1}(t)}{d t} .
\end{aligned}
$$

Because $E\left\{L f_{1}\left(X_{t}\right)\right\}=1$, we find $h_{1(t)}=t+\beta$ with $t \geq 0$. Additionally, $h_{1}(0)=E\left\{f_{1}\left(X_{0}\right)\right\}=0$ so that $\beta=0$. In other words, $h_{1}(t)=E\left\{f_{1}\left(X_{t}\right)\right\}=$ $t$.

When $k=2$ :

Let $h_{2}(t)=E\left\{f_{2}\left(X_{t}\right)\right\}$, we have $E\left\{L f_{2}\left(X_{t}\right)\right\}=$ $E\left\{f_{1}\left(X_{t}\right)\right\}=t$

so that $h_{2}(t)=\frac{t^{2}}{2}+\beta \quad$ for all $t \geq 0$. Moreover, $h_{2}(0)=E\left\{f_{2}\left(X_{0}\right)\right\}=0 \quad$ so $\quad$ we obtain $\beta=0$ and $h_{2}(t)=\frac{t^{2}}{2}$.

As a result, $E\left\{f_{k}\left(X_{t}\right)\right\}=\frac{t^{k}}{k}$ and $k=\overline{1,2}$ when $t$ is large enough. Expression (3.2) can be rewritten as the following form $E\left\{\frac{f_{k}\left(X_{t}\right)}{X_{t}^{k}} \times \frac{X_{t}^{k}}{t^{k}}\right\} \sim \frac{1}{k}$

when $t$ is large enough and for every $k=\overline{1,2}$.

Due to the existence of $\lim _{m \rightarrow \infty} f_{k}(m) / m^{k}$, the limit of $E\left\{\frac{x_{t}^{k}}{t^{k}}\right\}$ also exists. In what follows we evaluate $\lim _{m \rightarrow \infty} f_{k}(m) / m^{k}$ for $k=1,2$.

Lemma 3.2 For every $k=\overline{1,2}$, let $f_{k}$ be the function defined as in Proposition 3.1. Then we have $\lim _{m \rightarrow \pm \infty} \frac{f_{k}(m)}{m^{k}}=\frac{1}{k}\left(\frac{1}{\lambda(1-\rho)}\right)^{k}$.

Proof. We have

$$
\text { If } k=1 \text { then }
$$

$$
\lim _{m \rightarrow \pm \infty} \frac{f_{1}(m)}{m}=\lim _{m \rightarrow \pm \infty} \frac{m}{m \lambda(1-\rho)}=\frac{1}{\lambda(1-\rho)} .
$$

If $k=2:$ then 


$$
\begin{aligned}
\lim _{m \rightarrow \pm \infty} \frac{f_{2}(m)}{m^{2}}= & \lim _{m \rightarrow \pm \infty} \frac{1}{m^{2} \lambda^{2}(1-\rho)^{2}}\left(\frac{(m-1) m}{2}\right. \\
& \left.-\frac{m \rho}{1-\rho}\right)=\frac{1}{2}\left(\frac{1}{\lambda(1-\rho)}\right)^{2}
\end{aligned}
$$

This proves the lemma.

By Lemma 3.2, for every arbitrary constant $\epsilon>0$, there exists $M>0$ such that, for all $m \geq M$, we have

$$
\left|\frac{m^{2}}{f_{2}(m)}-2 G^{2}\right|<\epsilon .
$$

We recall that $G=\lambda(1-\rho)=\lambda-\gamma$. One has the following cases:

If $\left|X_{t}\right| \geq M$ then

$$
\begin{aligned}
\left|E\left(\left(\frac{X_{t}}{t}\right)^{2}-G^{2}\right)\right| & \approx\left|E\left(\frac{X_{t}^{2}}{t^{2}}-2 G^{2} \cdot \frac{f_{2}\left(X_{t}\right)}{t^{2}}\right)\right| \\
& \leq E\left(\left|\frac{X_{t}^{2}}{f_{2}\left(X_{t}\right)}-2 G^{2}\right| \cdot\left|\frac{f_{2}\left(X_{t}\right)}{t^{2}}\right|\right) \\
& \leq \frac{\epsilon}{2}
\end{aligned}
$$

When $t$ is large enough.

If $\left|X_{t}\right|<M$ then

$$
\begin{aligned}
\left|E\left(\left(\frac{X_{t}}{t}\right)^{2}-G^{2}\right)\right| & \approx\left|E\left(\frac{X_{t}^{2}}{t^{2}}-2 G^{2} \cdot \frac{f_{2}\left(X_{t}\right)}{t^{2}}\right)\right| \\
& \leq E\left(\frac{M^{2}}{t^{2}}+2 G^{2} \cdot \frac{\left|f_{2}\left(X_{t}\right)\right|}{t^{2}}\right) \leq \frac{D_{1}}{t^{2}}
\end{aligned}
$$

as $t$ is large enough. These estimations complete the proof.

Proposition 3.2 Let $\left(X_{t}\right)_{t \geq 0}$ be a stochastic process defined as above, we have $E\left(\frac{X_{t}}{t}-G\right)=O\left(t^{-1}\right)$.
Proof. For every arbitrary constant $\epsilon>0$, there exists $N>0$ such that, for all $m \geq N$, one has $\left|\frac{f_{1(m)}}{m}-\frac{1}{G}\right|<\epsilon$.

If $\left|X_{t}\right| \geq N$ then

$$
\begin{aligned}
\left|E\left(\frac{X_{t}}{t}-G\right)\right| & =\left|E\left(\frac{X_{t}}{t}-G \cdot \frac{f_{1}\left(X_{t}\right)}{t}\right)\right| \\
\leq & E\left(\left|\frac{X_{t}}{t} \cdot G\right| \cdot\left|\frac{1}{G}-\frac{f_{1}\left(X_{t}\right)}{X_{t}}\right|\right) \leq \epsilon
\end{aligned}
$$

When $t$ is large enough.

If $\left|X_{t}\right|<N$ then

$$
\begin{aligned}
\left|E\left(\frac{X_{t}}{t}-G\right)\right|=\mid & \left|E\left(\frac{X_{t}}{t}-G \cdot \frac{f_{1}\left(X_{t}\right)}{t}\right)\right| \\
& \leq E\left(\frac{N}{t}+G \cdot \frac{\left|f_{1}\left(X_{t}\right)\right|}{t}\right) \leq \frac{D_{2}}{t}
\end{aligned}
$$

when $t$ is large enough. We obtain the result.

\section{CONCLUSIONS}

The rate of convergence regarding the law of large numbers for the unbalanced stochastic process model in one dimensional space was evaluated. The method used in this paper can be applied in future research with other meaningful results.

\section{REFERENCES}

Depauw, J. and Derrien, J.M., 2009. Variance limite d'une marche aléatoire réversible en milieu aléatoire sur Z. Comptes Rendus Mathematique, 347(7-8): 401-406.

Lam, H.C., 2014. A quenched central limit theorem for reversible random walks in a random environment on Z. Journal of Applied Probability, 51(4): 1051-1064.

Ross, S. M., 2010. Introduction to Probability Models. Elsevier Inc, 782 pages. 